\title{
Cost of management of severe pneumonia in young children: systematic analysis
}

Shanshan Zhang ${ }^{1,2}$, Peter M. Sammon ${ }^{1}$, Isobel King ${ }^{1,3}$, Ana Lucia Andrade ${ }^{4}$, Cristiana M. Toscano ${ }^{4}$, Sheila N Araujo ${ }^{4,5}$, Anushua Sinha ${ }^{6}$, Shabir A. Madhi ${ }^{7,8}$, Gulam Khandaker, 10, Jiehui Kevin Yin ${ }^{9,10}$, Robert Booy, ${ }^{9,10}$, Tanvir M Huda ${ }^{11,12}$, Qazi S Rahman ${ }^{11}$, Shams El Arifeen ${ }^{11}$, Angela Gentile ${ }^{13}$, Norberto Giglio $^{13}$, Mejbah U. Bhuiyan ${ }^{14}$, Katharine Sturm-Ramirez ${ }^{14,15}$, Bradford D. Gessner ${ }^{16}$, Mardiati Nadjib ${ }^{17}$, Phyllis J. CarosoneLink $^{18}$, Eric AF Simões ${ }^{18,19}$, Jason A Child ${ }^{20}$, Imran Ahmed ${ }^{21}$, Zulfiqar A Bhutta ${ }^{21}$, Sajid B Soofi ${ }^{22}$, Rumana J Khan ${ }^{23}$, Harry Campbell ${ }^{1}$, Harish Nair ${ }^{1,24}$

${ }^{1}$ Usher Institute of Population Health Sciences and Informatics, University of Edinburgh, Edinburgh, UK

${ }^{2}$ Department of Preventive Dentistry, Peking University, School and Hospital of Stomatology, Beijing, PR China

${ }^{3}$ NHS Grampian, UK

${ }^{4}$ Department of Community Health, Federal University of Goias, Brazil

${ }^{5}$ State University of Maranhăo, Brazil

${ }^{6}$ New Jersey Medical School, Rutgers, The State University of New Jersey, Newark, New Jersey USA

Medical Research Council, Respiratory and Meningeal Pathogens Research Unit, Faculty of Health Sciences, University of the Witwatersrand,

Johannesburg, South Africa

${ }^{8}$ Department of Science and Technology/National Research Foundation,

Vaccine Preventable Diseases, University of the Witwatersrand,

Johannesburg, South Africa

${ }^{9}$ National Centre for Immunisation Research and Surveillance, The

Children's Hospital at Westmead, NSW, Australia

${ }^{10}$ Sydney School of Public Health, Faculty of Medicine, The University of Sydney, NSW, Australia

${ }^{11}$ Centre for Child and Adolescent Health, icddr,b, Dhaka, Bangladesh

${ }^{12}$ School of Public Health, Sydney Medical School, University of Sydney,

Sydney, Australia

${ }^{13}$ Epidemiology Department, Ricardo Gutierrez Children Hospital, University of Buenos Aires, Argentina

${ }^{14}$ Centre for Communicable Diseases, icddr,b, Dhaka, Bangladesh

${ }^{15}$ Influenza Division, National Center for Immunization and Respiratory

Diseases, Centers for Disease Control and Prevention, Atlanta, GA, USA

${ }^{16}$ Agence de Médicine Préventive, Paris, France

${ }^{17}$ Faculty of Public Health, University of Indonesia, Jakarta, Indonesia

${ }^{18}$ Department of Pediatrics, Section of Infectious Diseases, University of Colorado Denver School of Medicine, Denver, CO, USA

${ }^{19}$ Center for Global Health and Department of Epidemiology, Colorado School of Public Health, Aurora, CO, USA

${ }^{20}$ Pharmacy Department, Children's Hospital Colorado, Aurora, CO, USA

${ }^{21}$ Center of Excellence in Women and Child Health, the Aga Khan University, Karachi, Pakistan

${ }^{22}$ Department of Paediatrics \& Child Health, The Aga Khan University, Karachi, Pakistan

${ }^{23}$ James P Grant School of Public Health, BRAC University, Dhaka, Bangladesh

${ }^{24}$ Public Health Foundation of India, New Delhi, India

\section{Correspondence to:}

Dr Harish Nair

Centre for Global Health Research

Usher Institute of Population Health Sciences and Health Informatics

University of Edinburgh Medical School

Edinburgh EH8 9AG

United Kingdom

harish.nair@ed.ac.uk
Background Childhood pneumonia is a major cause of childhood illness and the second leading cause of child death globally. Understanding the costs associated with the management of childhood pneumonia is essential for resource allocation and priority setting for child health.

Methods We conducted a systematic review to identify studies reporting data on the cost of management of pneumonia in children younger than 5 years old. We collected unpublished cost data on non-severe, severe and very severe pneumonia through collaboration with an international working group. We extracted data on cost per episode, duration of hospital stay and unit cost of interventions for the management of pneumonia. The mean (95\% confidence interval, CI) and median (interquartile range, IQR) treatment costs were estimated and reported where appropriate.

Results We identified 24 published studies eligible for inclusion and supplemented these with data from 10 unpublished studies. The 34 studies included in the cost analysis contained data on more than 95000 children with pneumonia from both low- and-middle income countries (LMIC) and high-income countries (HIC) covering all $6 \mathrm{WHO}$ regions. The total cost (per episode) for management of severe pneumonia was US\$ 4.3 (95\% CI 1.5-8.7), US\$ 51.7 (95\% CI 17.491.0) and US\$2 242.7 (95\% CI 153.6-341.4)-559.4 (95\% CI 268.9-886.3) in community, out-patient facilities and different levels of hospital in-patient settings in LMIC. Direct medical cost for severe pneumonia in hospital inpatient settings was estimated to be $26.6 \%-115.8 \%$ of patients' monthly household income in LMIC. The mean direct non-medical cost and indirect cost for severe pneumonia management accounted for $0.5-31 \%$ of weekly household income. The mean length of stay (LOS) in hospital for children with severe pneumonia was 5.8 (IQR 5.3-6.4) and 7.7 (IQR 5.5-9.9) days in LMIC and HIC respectively for these children.

Conclusion This is the most comprehensive review to date of cost data from studies on the management of childhood pneumonia and these data should be helpful for health services planning and priority setting by national programmes and international agencies. 
Pneumonia is one of the leading causes of morbidity and mortality in children under-five globally, and accounted for about 935000 (15\%) deaths in 2013 and 120 million new episodes of illness in this age group in 2010 [1,2]. Appropriate management of childhood pneumonia can reduce pneumonia-specific mortality by $32-72 \%$ [3-5] and thus accelerate the progress toward achievement of the Millennium Development Goal 4 (MDG4). Childhood pneumonia places a large economic burden on families and the health care system, especially in resource-constrained low- and middle-income countries (LMIC). Severe ALRI is a substantial burden on health services worldwide and a major cause of hospital referral and admission in young children [6]. Although several studies in high-income as well as low- and middle-income countries have reported the costs associated with an episode of pneumonia (at the individual patient level), there are no published systematic reviews summarizing the evidence from different health systems and settings globally. Bahia et al reviewed pneumococcal disease costs and productivity loss in Latin America and the Caribbean showed variation in unit costs of pneumococcal pneumonia at outpatient and inpatient levels [7]. We aimed to conduct a systematic review of published data on the costs associated with management of pneumonia episodes in children younger than 5 years and to identify unpublished data sets from pneumonia research groups globally. Cost estimates based on these data should be useful to develop models for estimating cost of management of pneumonia in community as well as hospital-based settings.

\section{METHODS}

\section{Review of published studies}

We aimed to identify all published studies reporting empirical cost data on the treatment of episodes of pneumonia in children aged below 5 years during a 15-year period (1998-2013). We included studies in children younger than 5 years with pneumonia managed as in-patients or out-patients (using standard treatment per local standard) in secondary and tertiary hospitals, first level facility or in community settings. Data on the cost of a single episode of severe pneumonia from the societal and health care perspectives were collected as the primary study outcome. We developed a review protocol at the beginning of this study and followed the same throughout the process.

We undertook a systematic literature review with three reviewers (PS, IK, SZ), and hand searched reference list of all included articles. We searched four databases (with online search tools) to offer maximum coverage of the relevant literature: Medline, EMBASE, The Centre for Review and Dissemination Library (incorporating the DARE, NHS EED, and NHS HTA databases); and The Cochrane Library (via the Wiley Online Library) for the period 1 January 1998 to October 31 2013. (for search strategy, see Appendix S1 in Online Supplementary Document).

Three review authors (SZ, PS, IK) independently selected potentially relevant studies based on their title and abstract. Any disagreements in study selection or data extraction were resolved after discussion with SZ and HC. The eligible studies were retrieved electronically for full-text review. We included studies that investigated all-cause pneumonia in a non-selective population sample, reported empirical cost data for pneumonia treatment (using any intervention including, but not limited to, antibiotics), and included only children younger than 5 years or reported data separately for this age group. We excluded review articles, vaccine cost-effectiveness trials, and studies considering specially selected cohorts with severe co-morbidity (Appendix S2 in Online Supplementary Document). We developed and piloted a comprehensive data extraction template. We collected data on cost per episode, cost and unit cost of medication and services, duration of hospital stay and direct medical and non-medical costs. Direct medical cost included costs related to medication, diagnostic tests, medical staff time and hospital stay. Direct non-medical costs included those relating to food, transportation and accommodation charges. Any additional data on indirect costs such as care-givers' time and earning loss were also recorded, where available. Additionally, we extracted data on study characteristics including country, treatment setting, study type and sample size. We recorded the cost study perspective only if explicitly stated in the text of the article to avoid subjective influence. For those papers that did not explicitly state the perspective used, we noted "-" for "unstated".

We classified countries into high income and low-andmiddle income categories based on the classification adopted by the World Bank and according to 2012 Gross National Income (GNI) per capita, calculated using the World Bank Atlas method. The groups are low-income per capita US\$ 1035 or less; lower middle-income US\$ 1036-US\$ 4085; upper middle income US\$ 4086-US\$ 12615; and high income US $\$ 12616$ or more [8].

\section{Quality assessment}

We assessed the quality of the included studies using a 13 point scale based on a modified Drummond checklist [9] for economic evaluation focusing on the methodological robustness and detail of reporting (Appendix S3 in Online Supplementary Document). Studies were considered high quality if more than 10 points were addressed, medium quality studies covered 7-9 points and low quality studies addressed less than 6 points. Studies with all quality levels were included in the final analysis. 


\section{Unpublished data collection}

We collected unpublished data from 10 collaborating sites that were part of a Severe ALRI Working Group (SAWG) [6]. The study population included children under 5 years of age with a clinical diagnosis of pneumonia. We defined pneumonia using the World Health Organization's (WHO) Integrated Mangement of Childhood Illness (ICMI) definition by three different severity categories: non-severe, severe and very severe pneumonia based on WHO pocket book for hospital care for children 2005 [10]. We included all interventions for pneumonia management as detailed in the WHO pocket-book (for community/and facility-based management) where data were available.

We designed a costing spreadsheet with detailed descriptions of case definitions and methods and used this for data collection. Actual cost of medications, supplies, personnel and average laboratory costs were collected. Methods used to gather primary cost data in these studies were recorded in the spreadsheet. Resource utilization data from patient records were also documented, where available, including length of stay in hospital, the quantity of drugs and supplies utilized by each patient, and the use of diagnostic tests and procedures. We also attempted to collect data on outof-pocket spending (by patients) on transport and food where possible. Indirect cost of caregivers' time and daily pay rate were also recorded. Primary data collection was conducted using the provided standardized templates and guidelines at individual study site. (Appendix S4 in Online

\section{Supplementary Document).}

We used a bottom-up approach to calculate cost per episode for each level of the intervention (community, first level health facility and hospital). Costs were calculated and presented separately based on severity and service delivery channels: very severe pneumonia at hospital level (defined as pneumonia with central cyanosis, inability to breastfeed or drink, or vomiting everything, convulsions, lethargy or unconsciousness and severe respiratory distress diagnosed by doctor or physicians using WHO IMCI (2005) case definition or pneumonia cases requires critical care); severe pneumonia at hospital level (defined as pneumonia with chest indrawing using WHO IMCI definition or pneumo- nia need for hospital admission based on physician's assessment); severe pneumonia at community level (based on assessment by a trained health worker at home/first level facility using WHO IMCI (2005) case definition); and nonsevere pneumonia at outpatient level (defined as fast breathing for age in children aged 2 to 59 months). The costing model included direct medical cost, direct nonmedical cost and indirect costs. We calculated the cost per episode based on the estimates of the unit cost per contact (eg, unit cost of an antibiotic per day) at each management level multiplied by the resource utilization proportions (eg, $80 \%$ of children took amoxicillin for 5 days), plus indirect costs. For the mean total cost of treatment per episode we summed the cost of drugs, diagnostic investigations and hospital stay, as well as transportation and opportunity cost for caregivers' time. The formula is given in Figure $\mathbf{1}$.

We reported all cost data in 2013 US\$ equivalent prices. We first converted all costs to US $\$$ and then adjusted for inflation to 2013 values. Conversions were made using the Penn World Tables 8.0 (http://www.ggdc.net/pwt) and an online inflation-calculating tool (http://usinflation.org/cpiinflation-calculator) on 20 ${ }^{\text {th }}$ October 2013.

\section{Statistical analysis}

We have stratified the cost results by country income category: high-income countries (HIC) and low- and middleincome countries (LMIC). As an important input in the costing analysis for in-patient management, length of stay (LOS) in hospital was extracted for severe hospitalized cases. Cost per episode, cost by component (direct medical, direct nonmedical and indirect costs), and percentage of total cost per episode in each component were summarized. Cost per episode was synthesized by severity of diseases in each strata. The mean with $95 \% \mathrm{CI}$ of the treatment costs and the median with interquartile range (IQR) of LOS were estimated and reported where appropriate. The 95\% CIs were calculated based on 5000 bootstrap samples. Mean and median values were compared using appropriate statistical tests.

Direct medical cost in studies reported from household perspective were compared with monthly household income in respective countries to evaluate the burden on

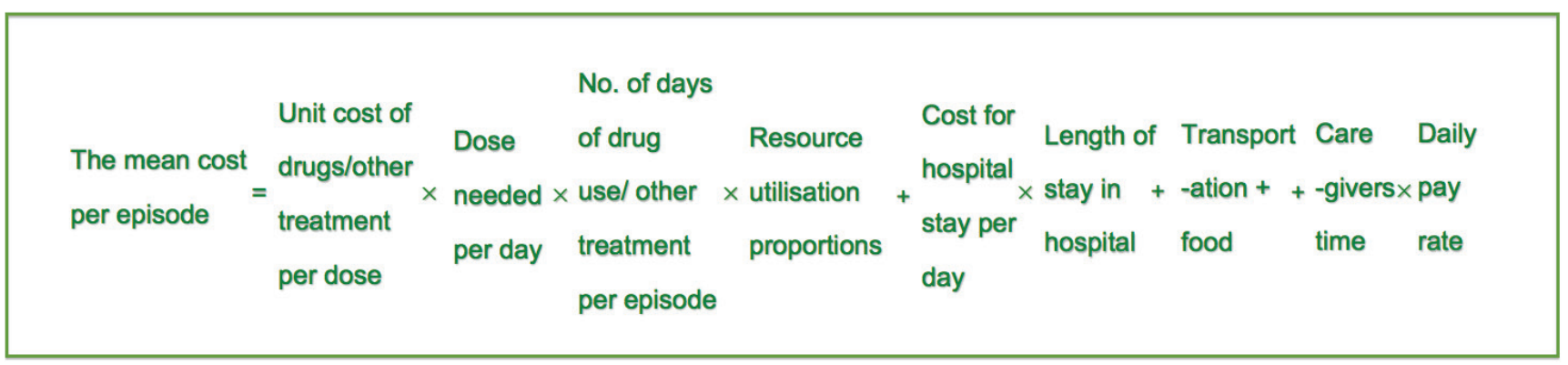

Figure 1. Formula for the mean total cost of treatment per episode. 
families. Monthly household incomes were derived from Gallup World Poll using annual median household income divided by 12 months [11]. These income results were based on Gallup data gathered between 2006 and 2012 in 131 populations. In two countries which annual household income data was missing, we used GNI per capita from World Bank database times the mean number of people per household instead. The percentages of direct non-medical costs and indirect cost per episode of weekly household income were also assessed to show the economic impact of pneumonia management for families when direct medical cost was not considered.

We conducted all data analyses using SPSS v. 19 (IBM, New York City, NY, USA) noting that included studies showed marked heterogeneity of population, methodology, treatment procedure reporting categories and perspectives.

\section{RESULTS}

\section{Search results}

We identified 789 studies through database searching, of which 60 articles were eligible for full text review on the basis of title and abstract assessment (Figure 2). Subsequently, only 24 papers were identified to be eligible for data extrac- tion and analysis. The key reasons for exclusions included: no data for children below 5 years or no cost data on pneumonia management were reported. For unpublished studies, we contacted 16 sites, 10 of which had data that met our eligibility criteria and contributed to the analysis. The unpublished cost data were for the period January 2001 to August 2012. Six of these sites provided cost data using a template and guidelines designed for this project while the remainder provided unpublished data in their own formats.

\section{Characteristics of published and unpublished data}

We identified 24 studies from the literature review and collected additional 10 data sets of unpublished studies totalling 34 studies from 21 countries across the 6 WHO regions (Table 1). Over $60 \%$ of the studies (21 out of 34) were conducted in the South East Asia and Africa Regions. The included studies reported data from a variety of treatment settings: community, out-patient and in-patient care settings in primary, secondary and tertiary hospitals, and at city, district, provincial and national levels. Twenty-seven of the 34 studies were stand-alone primary cost analysis and/or cost-of-illness studies. The remaining 7 studies were designed to collect cost data alongside clinical trials or epidemiological studies.

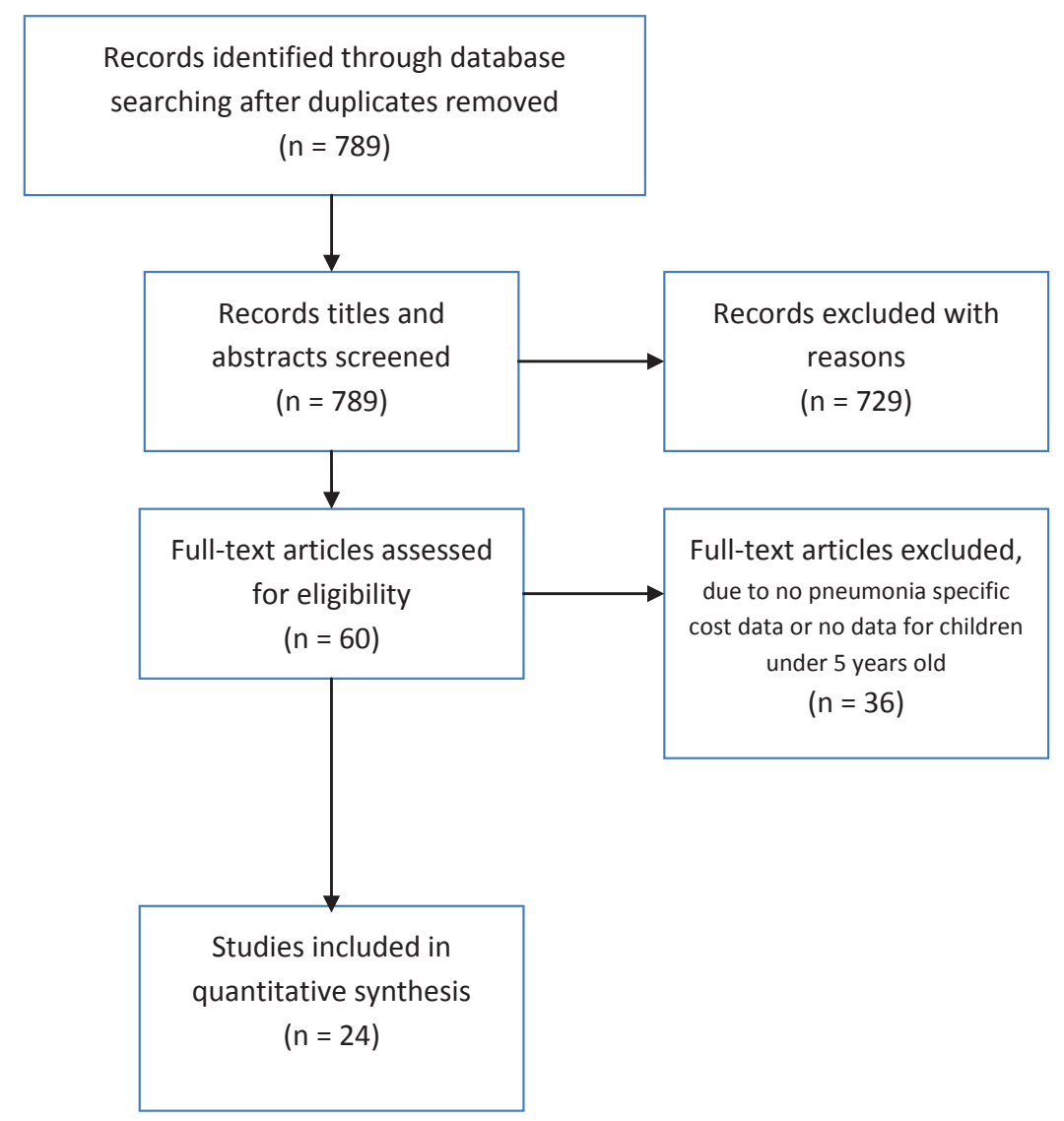

Figure 2. PRISMA flow diagram for severe pneumonia cost systematic review. 
Table 1. Characteristics of all studies included*

\begin{tabular}{|c|c|c|c|c|c|c|c|c|c|c|}
\hline $\begin{array}{l}\text { WHO } \\
\text { REGION }\end{array}$ & Countay, pubucation year & Study population & $\begin{array}{l}\text { HeALTHCARE } \\
\text { SETTING }\end{array}$ & $\begin{array}{l}\text { SEVERITY } \\
\text { OF } \\
\text { PMEUMONIA } \\
\text { STUDIED }\end{array}$ & Study DESIGN & $\begin{array}{l}\text { SourCE OF CASE } \\
\text { DEFINITION }\end{array}$ & Perspective & $\begin{array}{l}\text { SAMPLE } \\
\text { SIZE }\end{array}$ & $\begin{array}{l}\text { MeAN (SD) / } \\
\text { MEDIAN AGE } \\
\text { OF PATIENTS } \\
\text { (MONTHS) }\end{array}$ & $\begin{array}{l}\text { DATA } \\
\text { SOURGE }\end{array}$ \\
\hline \multicolumn{11}{|c|}{ High-income countries (number of studies $=8$ ) } \\
\hline \multirow[t]{3}{*}{ EUR } & $\begin{array}{l}\text { Northern Ireland, } \\
1999 \text { [39 }^{1}\end{array}$ & $\begin{array}{l}\text { Antrim } \\
\text { (urban) }\end{array}$ & $\mathrm{H} 2$ & $\mathrm{~S}$ & QES & $\mathrm{PD}$ & N/A- & 45 & $\begin{array}{l}39.60 \\
(16.8)\end{array}$ & $\mathrm{H}$ \\
\hline & Spain, 2013 [17] & $\begin{array}{l}\text { Barcelona } \\
\text { (urban) }\end{array}$ & H3 & S, VS & Cost analysis $\ddagger$ & $\begin{array}{l}\text { Culture-proved } \\
\text { pneumonia }\end{array}$ & Healthcare & 101 & 39.60 & $\mathrm{H}$ \\
\hline & Germany, 2005 [16] & National & $\mathrm{O}, \mathrm{Hl}$ & S, VS & Cost-of-illness & $\mathrm{PD}$ & Societal & 402 & N/A & $\mathrm{N}, \mathrm{IQ}$ \\
\hline \multirow[t]{2}{*}{ AMR } & $\begin{array}{l}\text { Chile, Uruguay, } \\
2007 \text { [12] }\end{array}$ & National & $\mathrm{O}, \mathrm{Hl}$ & S, NS & Cost analysis $\ddagger$ & PD, ICD-10 & Healthcare & 366 & N/A & $\mathrm{H}, \mathrm{IQ}$ \\
\hline & $\begin{array}{l}\text { United States, } \\
2012^{*}\end{array}$ & $\begin{array}{l}\text { Denver, } \\
\text { Colorado } \\
\text { (urban) }\end{array}$ & H3 & $\begin{array}{l}\text { S, VS, } \\
\text { NS }\end{array}$ & Cost-of-illness & $\begin{array}{l}\text { PD by WHO } \\
\text { IMCI definition }\end{array}$ & Societal & 940 & $0-59$ & $\mathrm{H}, \mathrm{P}$ \\
\hline \multirow[t]{3}{*}{ WPR } & Australia, 2008 [15] & National & $\mathrm{O}, \mathrm{Hl}-3$ & $\mathrm{~S}$ & Cost analysis $\ddagger$ & ICD-10 & Healthcare & 1348 & N/A & $\mathrm{N}$ \\
\hline & Australia, 2008 [14] & $\begin{array}{l}\text { Melbourne, } \\
\text { Victoria } \\
\text { (urban) }\end{array}$ & $\mathrm{O}, \mathrm{Hl}$ & $\mathrm{S}$ & $\begin{array}{l}\text { Cohort study/ } \\
\text { cost-of-illness }\end{array}$ & $\begin{array}{l}\text { Health } \\
\text { professional's } \\
\text { diagnosis }\end{array}$ & Societal & 528 & N/A & $\begin{array}{l}\mathrm{N}, \mathrm{H}, \mathrm{IQ}, \\
\text { Pilot }\end{array}$ \\
\hline & Australia, 2011* & $\begin{array}{l}\text { Sydney } \\
\text { (urban) }\end{array}$ & $\mathrm{H} 3$ & S, VS & Cost-of-illness & $\begin{array}{l}\text { PD by WHO } \\
\text { IMCI definition }\end{array}$ & Societal & N/A & N/A & $\begin{array}{l}\mathrm{P}, \\
\text { Market } \\
\text { price }\end{array}$ \\
\hline \multicolumn{11}{|c|}{ Low- and middle-income countries (number of studies $=27$ ) } \\
\hline \multirow[t]{15}{*}{ SEAR } & $\begin{array}{l}\text { Bangladesh, } 2010 \\
{[26]}\end{array}$ & Dhaka (urban) & H3 & $\mathrm{S}$ & Cost-of-illness & $\mathrm{PD}$ & Family & 90 & 5.00 & IQ \\
\hline & Bangladesh, $2005 \dagger$ & Dhaka (urban) & H3 & $\mathrm{S}, \mathrm{VS}$ & Cost-of-illness & $\begin{array}{l}\text { PD by WHO } \\
\text { IMCI definition }\end{array}$ & Household & 114 & 70.32 & IQ \\
\hline & $\begin{array}{l}\text { Bangladesh, } 2010 \\
\text { [24] }\end{array}$ & $\begin{array}{l}\text { Mirpur, Dhaka } \\
\text { (urban) }\end{array}$ & $\mathrm{O}, \mathrm{H} 2$ & $\mathrm{~S}$ & RCT/CEA & $\begin{array}{l}\text { PD by WHO } \\
\text { IMCI definition }\end{array}$ & Societal & 360 & 8.00 & - \\
\hline & Bangladesh, $2010 \dagger$ & $\begin{array}{l}\text { Barishal, } \\
\text { Bogra, } \\
\text { Comilla, } \\
\text { Kishoregonj } \\
\text { (urban) }\end{array}$ & H3 & S, NS & Cost-of-illness & $\begin{array}{l}\text { PD by WHO } \\
\text { IMCI definition }\end{array}$ & Societal & 235 & N/A & IQ \\
\hline & Bangladesh, $2012 \dagger$ & $\begin{array}{l}\text { Mohakhali, } \\
\text { Dhaka (urban) }\end{array}$ & H3 & $\begin{array}{l}\text { S, VS, } \\
\text { NS }\end{array}$ & Cost-of-illness & $\begin{array}{l}\text { PD by WHO } \\
\text { IMCI definition }\end{array}$ & Societal & 340 & N/A & $\mathrm{H}$ \\
\hline & India, 2009 [30] & Vellore (rural) & $\mathrm{H} 1, \mathrm{H} 2$ & $\mathrm{~S}$ & Cost-of-illness & $\begin{array}{l}\text { PD by WHO } \\
\text { IMCI definition }\end{array}$ & $\begin{array}{l}\text { Healthcare/ } \\
\text { Household }\end{array}$ & 56 & 8.8 & $\mathrm{H}, \mathrm{IQ}$ \\
\hline & India, 2002 [29] & $\begin{array}{l}\text { Berhampur, } \\
\text { Orissa (urban } \\
\text { and rural) }\end{array}$ & H3 & $\mathrm{S}$ & $\begin{array}{l}\text { Epidemiologi- } \\
\text { cal study }\end{array}$ & $\mathrm{PD}$ & Societal & 52 & N/A & $\mathrm{H}, \mathrm{IQ}$ \\
\hline & Indonesia, $2001 \dagger$ & $\begin{array}{l}\text { Lombok } \\
\text { (rural) }\end{array}$ & H3 & $\mathrm{S}$ & Cost-of-illness & $\begin{array}{l}\text { PD by WHO } \\
\text { IMCI definition }\end{array}$ & Societal & N/A & N/A & $\mathrm{H}$ \\
\hline & Pakistan, 2003 [25] & $\begin{array}{l}\text { Peshawar city } \\
\text { (urban) }\end{array}$ & H3 & $\mathrm{S}$ & RCT/CEA & $\begin{array}{l}\text { PD by WHO } \\
\text { IMCI definition }\end{array}$ & - & 126 & N/A & - \\
\hline & Pakistan, 2006 [20] & $\begin{array}{l}\text { Ghizer district } \\
\text { (rural) }\end{array}$ & $\begin{array}{l}\mathrm{O}, \mathrm{H} 1 \\
\mathrm{H} 2\end{array}$ & S, NS & Cost analysis $\ddagger$ & $\mathrm{PD}$ & Societal & 502 & N/A & IQ \\
\hline & Pakistan, 2008 [19] & $\begin{array}{l}\text { Ghizer district } \\
\text { (rural) }\end{array}$ & $\begin{array}{l}\mathrm{O}, \mathrm{H} 1, \\
\mathrm{H} 2\end{array}$ & $\begin{array}{l}\text { S, VS, } \\
\text { NS }\end{array}$ & Cost analysis $\ddagger$ & $\begin{array}{l}\text { PD by WHO } \\
\text { IMCI definition }\end{array}$ & Healthcare & 141 & N/A & IQ \\
\hline & Pakistan, $2010^{\dagger}$ & Matiari (rural) & C & $\mathrm{S}$ & Cost-of-illness & $\begin{array}{l}\text { PD by WHO } \\
\text { IMCI definition }\end{array}$ & Healthcare & N/A & N/A & $\begin{array}{l}\text { Surveil- } \\
\text { lance }\end{array}$ \\
\hline & Pakistan, 2012 [23] & $\begin{array}{l}\text { Haripur } \\
\text { district (rural) }\end{array}$ & $\mathrm{C}, \mathrm{H} 1, \mathrm{H} 2$ & $\mathrm{~S}$ & Cost analysis $\ddagger$ & $\begin{array}{l}\text { WHO } \\
\text { definition by } \\
\text { health worker }\end{array}$ & Household & 423 & N/A & $\mathrm{H}, \mathrm{IQ}$ \\
\hline & Viet Nam, 2010 [18] & $\begin{array}{l}\text { Nha Trang city } \\
\text { (urban) }\end{array}$ & $\mathrm{H} 2$ & $\begin{array}{l}\text { S, VS, } \\
\text { NS }\end{array}$ & Cost-of-illness & $\begin{array}{l}\text { PD by WHO } \\
\text { IMCI definition }\end{array}$ & Healthcare & 788 & 12.67 & $\mathrm{~N}, \mathrm{H}$ \\
\hline & Viet Nam, 2001 [28] & $\begin{array}{l}\text { Ba Vi district } \\
\text { (rural) }\end{array}$ & $\mathrm{C}, \mathrm{O}, \mathrm{Hl}$ & $\mathrm{S}$ & Cost analysis $\ddagger$ & $\begin{array}{l}\text { WHO } \\
\text { definition, } \\
\text { self-reported }\end{array}$ & Household & 94 & N/A & IQ \\
\hline
\end{tabular}


Table 1. Continued

\begin{tabular}{|c|c|c|c|c|c|c|c|c|c|c|}
\hline $\begin{array}{l}\text { WHO } \\
\text { REGION }\end{array}$ & Counthy, Publication year & Study population & $\begin{array}{l}\text { HeALTHCARE } \\
\text { SETtING }\end{array}$ & $\begin{array}{l}\text { SEVERITY } \\
\text { OF } \\
\text { PMEUMONIA } \\
\text { STUDIED }\end{array}$ & Study Design & $\begin{array}{l}\text { Source OF CASE } \\
\text { DEFINIIIION }\end{array}$ & Perspective & $\begin{array}{l}\text { SAMPLE } \\
\text { SIZE }\end{array}$ & $\begin{array}{l}\text { MeAn (SD) / } \\
\text { MEDIAN AGE } \\
\text { OF PATIENTS } \\
\text { (MONTHS) }\end{array}$ & $\begin{array}{l}\text { DATA } \\
\text { SOURCE }\end{array}$ \\
\hline \multirow[t]{6}{*}{ AFR } & Guinea, 1998 [21] & National & $\mathrm{O}, \mathrm{Hl}$ & S, NS & CEA & PD & - & 73650 & N/A & $\mathrm{H}, \mathrm{E}$ \\
\hline & $\begin{array}{l}\text { South Africa, } 2011 \\
\text { [33] }\end{array}$ & $\begin{array}{l}\text { Pretoria } \\
\text { (urban) }\end{array}$ & H3 & S, VS & Cost analysis $\neq$ & $\begin{array}{l}\text { WHO } \\
\text { definition }\end{array}$ & - & 3014 & N/A & $\mathrm{H}$ \\
\hline & $\begin{array}{l}\text { South Africa, } 2012 \\
{[22]}\end{array}$ & National & H3 & $\begin{array}{l}\text { S, VS, } \\
\text { NS }\end{array}$ & RCT & $\mathrm{PD}$ & $\begin{array}{l}\text { Societal/ } \\
\text { health care }\end{array}$ & 745 & N/A & H, IQ \\
\hline & South Africa, $2001 \dagger$ & $\begin{array}{l}\text { Soweto } \\
\text { (urban) }\end{array}$ & H3 & S, VS & Cost-of-illness & $\begin{array}{l}\text { PD by WHO } \\
\text { IMCI definition }\end{array}$ & Societal & 509 & 14.00 & $\mathrm{H}, \mathrm{IQ}$ \\
\hline & Kenya, 2009 [32] & National & $\begin{array}{l}\mathrm{H} 3, \mathrm{H} 2, \\
\mathrm{H} 1\end{array}$ & $\mathrm{~S}$ & Cost analysis $\ddagger$ & $\mathrm{PD}$ & Societal & 205 & 12.00 & $\mathrm{H}, \mathrm{IQ}$ \\
\hline & Zambia, 2009 [31] & $\begin{array}{l}\text { Kanyama } \\
\text { Township } \\
\text { (urban) }\end{array}$ & $\mathrm{O}, \mathrm{H} 2$ & $\mathrm{~S}$ & Cost analysis $\ddagger$ & $\mathrm{PD}$ & Healthcare & 9146 & N/A & $\mathrm{N}, \mathrm{H}, \mathrm{P}, \mathrm{W}$ \\
\hline \multirow[t]{4}{*}{ AMR } & $\begin{array}{l}\text { Colombia, } 2013 \\
{[27]}\end{array}$ & National & $\mathrm{H} 1, \mathrm{H} 2, \mathrm{H} 3$ & $\begin{array}{l}\text { S, VS, } \\
\text { NS }\end{array}$ & $\begin{array}{l}\text { Cost-of-ill- } \\
\text { ness§ }\end{array}$ & $\begin{array}{l}\text { WHO } \\
\text { definition, } \\
\text { radiographical- } \\
\text { ly diagnosed }\end{array}$ & Healthcare & 1545 & N/A & I \\
\hline & Brazil, 2011† & $\begin{array}{l}\text { Goiânia } \\
\text { (urban) }\end{array}$ & H3 & S, VS & $\begin{array}{l}\text { Cost-of-ill- } \\
\text { ness§ }\end{array}$ & $\begin{array}{l}\text { PD by WHO } \\
\text { IMCI definition }\end{array}$ & Societal & 79 & $0-36$ & $\mathrm{H}, \mathrm{N}$ \\
\hline & Argentina, $2012 \dagger$ & $\begin{array}{l}\text { Buenos Aires } \\
\text { (urban) }\end{array}$ & H3 & S, VS & $\begin{array}{l}\text { Cost-of-ill- } \\
\text { ness§ }\end{array}$ & $\begin{array}{l}\text { PD by WHO } \\
\text { IMCI definition }\end{array}$ & Societal & N/A & N/A & $\mathrm{N}$ \\
\hline & Brazil,2007 [12] & National & $\mathrm{O}, \mathrm{Hl}$ & S, NS & Cost analysis $\ddagger$ & PD, ICD-10 & Healthcare & 366 & N/A & $\mathrm{H}, \mathrm{IQ}$ \\
\hline WPR & Fiji, 2012 [34] & $\begin{array}{l}\text { Viti Levu } \\
\text { (urban and } \\
\text { rural) }\end{array}$ & $\mathrm{O}$ & $\mathrm{S}$ & Cost analysis $\ddagger$ & $\begin{array}{l}\text { PD by WHO } \\
\text { IMCI definition }\end{array}$ & $\begin{array}{l}\text { Societal/ } \\
\text { household }\end{array}$ & 390 & N/A & $\mathrm{N}, \mathrm{H}, \mathrm{IQ}$ \\
\hline EMR & Jordan, 2010 [35] & Amman & $\mathrm{Hl}$ & $\mathrm{S}$ & Cohort study & $\mathrm{PD}$ & - & 728 & 4.30 & N/A \\
\hline
\end{tabular}

* Severity of pneumonia: NS - non severe, S - severe, VS - very severe. Data source: H - hospital records, N - national data, IQ - interviews and questionnaires, I - insurance database, P - pharmacy database, W - WHO database. Treatment settings: H3 - tertiary hospital in-patient, H2-secondary hospital in-patient, $\mathrm{Hl}$ - primary hospital inpatient, $\mathrm{O}$ - out-patient care, C - community ambulatory care; PD - physician's diagnosis, CEA - cost effectiveness analysis, RCT - randomized clinical trial, QES- quasi-experimental study, N/A - not available, PD - physician's diagnosis, IMCI - Integrated Management of Childhood Illness, WHO - World Health Organization, SD - standard deviation.

EUR - Europe Region, AMR - the Americas Region, WPR - Western Pacific Region, SEAR - South East Asia, AFR- The Africa Region, EMR- Eastern Mediterranean Region

$\dagger$ Unpublished data.

¥The analysis of the comparative costs of alternative treatments or health care programmes.

$\S$ The cost analysis of treatment of a disease.

The included studies reported cost data on a total of 97062 children treated at facility or community levels, with a median sample size of 378 (IQR 117-741) across all studies. The age of the participants was reported in 12 studies and the median age was 12.3 months (IQR 8.20-33.20). The perspective of costing was explicitly stated in 30 of 34 studies. Of these, the most common perspectives were societal (16 out of 33, 1 study did not specify perspective), health care (11 out of 33) and household (5 out of 33). In most studies, the sources of pneumonia case definition were physician's diagnosis according to WHO IMCI definition (29 out of 34), culture-proved pneumonia was used for case definition in 1 study, self-reported condition according to WHO IMCI definition was adopted in one study, and two studies used health workers' diagnosis. A number of different sources were used for gathering cost data, the most common being through hospital records and costing interviews/questionnaires. Interviews and questionnaires were commonly used in studies with a household perspective to collect data on indirect costs. Other sources included a national database of costs, insurance databases, surveillance data and pharmaceutical databases. The WHO CHOICE database, expert opinion and data from pilot studies were also used to collect data on the unit cost of pneumonia treatment.

The average quality score of 24 published studies was 8.21 out of 13 on scale based on modified Drummond checklist (range 3-12) (Appendix S5 in Online Supplementary Document). The majority of the studies failed to consider discounting and did not perform sensitivity analyses. There were 6 studies considered high quality, 14 studies were medium quality, and 4 low quality studies. All studies were included into the final analysis. 


\section{Cost of management per episode of pneumonia}

Cost results stratified by income category are presented in Table 2, Table 3 and Figure 3. In HICs, the mean cost of treatment for an episode of severe pneumonia at the hospital out-patient level was US\$251.1 in Germany [16]. An Australian study [14] reported similar cost of US\$ 254.9 for community management of severe pneumonia. Average costs of facility based case management for young children admitted in primary/sary and tertiary hospitals were US\$ 2803.5 (95\% CI IQR 2000.6-3683.3), and US\$ 7037.2 (95\% CI 4028.6-11311.0) respectively, which was 11-28 fold higher than in those managed as out-patients. The total cost per episode for the management of non-severe pneumonia at out-patient level was also reported for three countries: US\$55.8 in Uruguay [12], US\$272.7 in Chile [12] and US\$ 334.6 in the United States [36]. The cost for very severe pneumonia managed in general pediatric wards followed by intensive care unit (ICU) care was reported to be US\$9151.3 in a tertiary hospital in Spain [17] and US\$ 120576.3 in the United States, which is nearly 2-17 fold of the cost for severe pneumonia management in hospital settings in HICs. The majority of studies from HIC took only direct medical costs into consideration. Only two studies included direct non-medical costs and indirect costs [16]. The mean proportion of the total cost for direct medical, direct non-medical and indirect costs were $41.5 \%, 19.5 \%$ and $38.5 \%$ respectively.

In LMICs, the cost of case management for severe pneumonia was reported across all treatment settings. The community management cost was only reported in studies conducted in South-East Asia region, with a mean cost of US\$ 4.3 (95\% CI 1.5-8.7) per episode. Out-patient care mean costs were US\$ 51.7 (95\% CI 17.4-91.0) per case. Costs for inpatient care varied by regions, level of hospitals (primary/ sary/tertiary), and levels of care offered at a facility: the mean cost for primary/sary hospital care was 242.7 (95\% CI 153.6-341.4) and for tertiary/teaching hospital was 559.4 (95\% CI 268.9-886.3). Two groups-severe pneumonia by WHO IMCI definition and hospitalized pneumonia by physician's diagnosis-showed similar costs in all levels of care. The in-patient care costs were 4-11 fold greater than that for out-patient care in the LMICs strata, which in turn was significantly higher than that for community management.

Table 2. Cost per episode for childhood pneumonia management in high-income countries

\begin{tabular}{|c|c|c|c|c|c|c|c|c|c|c|c|}
\hline \multirow[t]{2}{*}{ SEVERITY } & \multirow[t]{2}{*}{$\begin{array}{l}\text { WHO } \\
\text { REGION }\end{array}$} & \multirow[t]{2}{*}{ COUNTRY, PUBulcation year } & \multirow[t]{2}{*}{ Perspective } & \multirow[t]{2}{*}{$\begin{array}{l}\text { SAMPLE } \\
\text { SIZE }\end{array}$} & \multicolumn{4}{|c|}{ Cost per EPISODE (2013 US\$) } & \multicolumn{3}{|c|}{$\begin{array}{l}\text { COST COMPONENT, \% OF TOTAL } \\
\text { COST PER EPISODE }\end{array}$} \\
\hline & & & & & $\begin{array}{c}\text { Tertiary/teaching } \\
\text { hospital in-patient } \\
\text { care }\end{array}$ & $\begin{array}{c}\text { Secondary/ } \\
\text { primary } \\
\text { hospital } \\
\text { in-patient care }\end{array}$ & $\begin{array}{l}\text { Out- } \\
\text { patient } \\
\text { care }\end{array}$ & $\begin{array}{l}\text { Com- } \\
\text { muni- } \\
\text { ty care }\end{array}$ & $\begin{array}{l}\text { Di- } \\
\text { rect } \\
\text { med- } \\
\text { ical }\end{array}$ & $\begin{array}{c}\text { Direct } \\
\text { non- } \\
\text { medical }\end{array}$ & $\begin{array}{r}\text { Indi- } \\
\text { rect }\end{array}$ \\
\hline \multirow{3}{*}{$\begin{array}{l}\text { Non-severe } \\
\text { pneumonia }\end{array}$} & \multirow[t]{3}{*}{ AMR } & US, $2012 *$ & Societal & 940 & & & 334.6 & & & & \\
\hline & & Chile, 2007 [12] & Healthcare & 366 & & & 272.7 & & & & \\
\hline & & Uruguay, 2007 [12] & Healthcare & 366 & & & 55.8 & & & & \\
\hline \multicolumn{5}{|c|}{ Non-severe pneumonia mean cost $(95 \% \mathrm{CI})$} & \multicolumn{7}{|c|}{$221.0(55.8-334.6)$} \\
\hline \multirow{5}{*}{$\begin{array}{l}\text { Severe } \\
\text { pneumonia } \\
\text { by WHO } \\
\text { IMCI } \\
\text { Definition }\end{array}$} & AMR & Chile, 2007 [12] & Healthcare & 366 & & 4316.7 & & & 100 & & \\
\hline & & Uruguay, 2007 [12] & Healthcare & 366 & & 1421.6 & & & 100 & & \\
\hline & & US, $2012 *$ & Societal & 940 & 15029.2 & & & & & & \\
\hline & EUR & $\begin{array}{l}\text { North Ireland, } 1999 \\
\text { [13] }\end{array}$ & $\mathrm{NA}$ & 45 & $5733.8,2716.8$ & & & & 100 & & \\
\hline & WPR & Australia, 2011* & Societal & NA & $6,259.1$ & & & & 93.1 & & 6.9 \\
\hline \multirow{4}{*}{$\begin{array}{l}\text { Hospitalised } \\
\text { pneumonia }\end{array}$} & WPR & Australia, 2008 [14] & Societal & 528 & & 2813.1 & & 254.9 & 100 & & \\
\hline & & Australia, 2008 [15] & Healthcare & 1348 & & 2307.8 & & & 100 & & \\
\hline & EUR & Germany, 2005 [16] & Societal & 402 & & 3158.6 & 251.1 & & 41.5 & 19.5 & 38.5 \\
\hline & & Spain, 2013 [17] & Healthcare & 101 & 5447.3 & & & & 100 & & \\
\hline \multicolumn{5}{|c|}{ Severe pneumonia mean cost $(95 \% \mathrm{CI})$} & $\begin{array}{c}7037.2 \\
(40286-11311.0) \\
\end{array}$ & $\begin{array}{c}2803.5 \\
(2000.6-3683.3) \\
\end{array}$ & 251.1 & 254.9 & & & \\
\hline $\begin{array}{l}\text { Very severe } \\
\text { pneumonia } \\
\text { by IMCI }\end{array}$ & AMR & US, 2012* & Societal & 940 & 120576.2 & & & & & & \\
\hline $\begin{array}{l}\text { Very severe } \\
\text { pneumonia } \\
\text { requiring } \\
\text { critical care }\end{array}$ & AMR & Spain, 2013 [17] & Healthcare & 101 & 9151.3 & & & & & & \\
\hline
\end{tabular}

Very severe pneumonia mean cost (95\% CI) 64863.8 (9151.3-120576.3)

NA - Information not available, EUR - Europe Region, AMR - The Americas Region, WPR - Western Pacific Region, CI - confidence interval, IMCI Integrated Management of Childhood Illness

*Unpublished data. 
Table 3. Cost per episode for childhood pneumonia management in low- and middle-income countries

\begin{tabular}{|c|c|c|c|c|c|c|c|c|c|c|c|}
\hline \multirow[t]{2}{*}{ SEVERITY } & \multirow[t]{2}{*}{$\begin{array}{l}\text { WHO } \\
\text { REGION }\end{array}$} & \multirow[t]{2}{*}{ Country, Publication year } & \multirow[t]{2}{*}{ Perspective } & \multirow[t]{2}{*}{$\begin{array}{l}\text { SAMPLE } \\
\text { SIZE }\end{array}$} & \multicolumn{4}{|c|}{ CoSt PER EPISODE (2013 USS) } & \multicolumn{3}{|c|}{$\begin{array}{l}\text { Cost COMPONENT, \% OF } \\
\text { TOTAL COST PER EPISODE }\end{array}$} \\
\hline & & & & & $\begin{array}{c}\text { Tertiary/teaching } \\
\text { hospital } \\
\text { in-patient care }\end{array}$ & $\begin{array}{c}\text { Secondary/ } \\
\text { primary } \\
\text { hospital } \\
\text { in-patient care }\end{array}$ & $\begin{array}{l}\text { Out-patient } \\
\text { care }\end{array}$ & $\begin{array}{l}\text { Commu- } \\
\text { nity care }\end{array}$ & $\begin{array}{l}\text { Direct } \\
\text { medi- } \\
\text { cal }\end{array}$ & $\begin{array}{c}\text { Direct } \\
\text { non- } \\
\text { medi- } \\
\text { cal }\end{array}$ & $\begin{array}{l}\text { In- } \\
\text { di- } \\
\text { rect }\end{array}$ \\
\hline \multirow{7}{*}{$\begin{array}{l}\text { Non-severe } \\
\text { pneumonia }\end{array}$} & SEAR & Viet Nam, 2010 [18] & Healthcare & 788 & & & 28.6 & & & & \\
\hline & & Pakistan, 2008 [19] & Healthcare & 141 & & & 29.4 & & & & \\
\hline & & Pakistan, 2006 [20] & Societal & 502 & & & $94.1-17.8$ & & & & \\
\hline & & Bangladesh, 2012* & Societal & 340 & & & 5.7 & & & & \\
\hline & AFR & Guinea, 1998 [21] & NA & 73650 & & & 3.2 & & & & \\
\hline & & South Africa, 2012 [22] & $\begin{array}{l}\text { Societal/ } \\
\text { health care }\end{array}$ & 745 & & & 263.1 & & & & \\
\hline & AMR & Brazil, 2007 [12] & Healthcare & 366 & & & 93.0 & & & & \\
\hline \multicolumn{5}{|c|}{ Non-severe pneumonia mean cost $(95 \% \mathrm{CI})$} & \multicolumn{7}{|c|}{$66.9(21.7-129.7)$} \\
\hline \multirow{16}{*}{$\begin{array}{l}\text { Severe } \\
\text { pneumonia } \\
\text { by WHO } \\
\text { IMCI } \\
\text { Definition }\end{array}$} & SEAR & Pakistan, 2010* & Healthcare & NA & & & & 8.7 & 100 & & \\
\hline & & Pakistan, 2012 [23] & Household & 423 & & & 7.9 & 1.5 & 89.1 & 1.3 & 9.6 \\
\hline & & Bangladesh, 2012* & Societal & 340 & & & 5.7 & & & & \\
\hline & & Bangladesh, 2010 [24] & Societal & 360 & 193.6 & & 124.0 & & $\mathrm{Y}$ & $\mathrm{Y}$ & \\
\hline & & Viet Nam, 2010 [18] & Healthcare & 788 & & 39.5 & & & $\mathrm{Y}$ & $\mathrm{Y}$ & $\mathrm{Y}$ \\
\hline & & Pakistan, 2008 [19] & Healthcare & 141 & & 186.0 & & & 64.1 & 35.9 & \\
\hline & & Pakistan, 2003 [25] & $\mathrm{NA}$ & 126 & 20.3 & & & & 100 & & \\
\hline & & Bangladesh, 2005* & Household & 114 & $80.6 \dagger$ & $62.6 \#$ & & & $70.9 \dagger$ & $29.1 \dagger$ & \\
\hline & & Bangladesh, 2010 [26] & Household & 90 & 124.2 & & & & 67.6 & 32.4 & \\
\hline & & Indonesia, 2001* & Societal & NA & 135.2 & & & & 75 & 25 & \\
\hline & AFR & Guinea, 1998 [21] & $\mathrm{NA}$ & 73650 & & 110.6 & & & 69 & 30 & \\
\hline & & South Africa, 2001* & Societal & 509 & 480.98 & 110.0 & & & & & \\
\hline & AMR & Brazil, 2007 [12] & Healthcare & 366 & & 461.0 & & & 100 & & \\
\hline & & Brazil, 2011* & Societal & 79 & $1474.1 \dagger, \ddagger$ & 594.5\# & & & $94 \dagger$ & $1 \dagger$ & $5 \dagger$ \\
\hline & & Colombia, 2013[27] & Healthcare & 1545 & & 517.6 & & & 100 & & \\
\hline & & Argentina, 2012* & Societal & NA & 1648.0 & & & & 100 & & \\
\hline \multirow{11}{*}{$\begin{array}{l}\text { Hospitalised } \\
\text { pneumonia }\end{array}$} & SEAR & Viet Nam, 2001 [28] & Household & 94 & & & & 2.7 & $56-88$ & $\mathrm{Y}$ & \\
\hline & & Pakistan, 2006 [20] & Societal & 502 & & 310.8 & 127.6 & & 45.3 & 55 & \\
\hline & & India, 2009 [29] & $\begin{array}{l}\text { Healthcare/ } \\
\text { household }\end{array}$ & 56 & 145.7 & 44.7 & & & 45.7 & 5.3 & 47.4 \\
\hline & & India, 2002 [30] & Societal & 52 & 23.9 & & & & 100 & & \\
\hline & AFR & Zambia, 2009 [31] & Healthcare & 9146 & & 249.7 & 55.7 & & 100 & & \\
\hline & & Kenya, 2009 [32] & Societal & 205 & 236.8 & $162.1,89.5$ & & & 86 & 14 & $\bar{Y}$ \\
\hline & & South Africa, 2011 [33] & Societal & 509 & $491.4 \dagger, 1553.2 \ddagger$ & & & & 100 & & \\
\hline & & South Africa, 2012 [22] & $\begin{array}{l}\text { Societal/ } \\
\text { health care }\end{array}$ & 745 & 1223.1 & & & & 98 & 2 & 0.2 \\
\hline & $\overline{W P R}$ & Fiji, 2012 [34] & $\begin{array}{l}\text { Societal/ } \\
\text { household }\end{array}$ & 390 & & & $25.7,15.6$ & & 61.9 & 33.2 & 4.9 \\
\hline & AMR & Colombia, 2013 [27] & Healthcare & 1545 & & 304.4 & 76.2 & & & & \\
\hline & EMR & Jordan, $2012 *$ & $\mathrm{NA}$ & 728 & & 563.4 & & & 100 & & \\
\hline \multicolumn{4}{|c|}{ Severe pneumonia mean cost $(95 \% \mathrm{CI})$} & & $\begin{array}{c}559.4 \\
(268.9-886.3) \\
\end{array}$ & $\begin{array}{c}242.7 \\
(153.6-341.4) \\
\end{array}$ & $\begin{array}{c}51.7 \\
(17.4-91.0) \\
\end{array}$ & $\begin{array}{c}4.3 \\
(1.5-8.7) \\
\end{array}$ & & & \\
\hline \multirow{3}{*}{$\begin{array}{l}\text { Very severe } \\
\text { pneumonia } \\
\text { by IMCI }\end{array}$} & SEAR & Bangladesh, 2012* & Societal & 340 & & & 15.7 & & & & \\
\hline & & Viet Nam, 2010 [18] & Healthcare & 788 & & 61.2 & & & & & \\
\hline & & Pakistan, 2008 [19] & Healthcare & 141 & & 81.3 & & & & & \\
\hline \multirow{3}{*}{$\begin{array}{l}\text { Very severe } \\
\text { pneumonia } \\
\text { requiring } \\
\text { critical care }\end{array}$} & AFR & South Africa, 2011 [33] & NA & 3014 & $\begin{array}{c}849.0 \dagger \\
14795.4 \neq\end{array}$ & & & & & & \\
\hline & & South Africa, 2012 [22] & $\begin{array}{l}\text { Societal/ } \\
\text { health care }\end{array}$ & 745 & 6696.2 & & & & & & \\
\hline & AMR & Colombia [27] & Healthcare & 1545 & 3643.4 & & & & & & \\
\hline \multicolumn{5}{|c|}{ Very severe pneumonia mean cost $(95 \% \mathrm{CI})$} & $\begin{array}{c}6496.0 \\
(2246.2-12007.4) \\
\end{array}$ & $\begin{array}{c}71.3 \\
(61.2-81.3) \\
\end{array}$ & $\begin{array}{c}15.7 \\
(15.7-15.7) \\
\end{array}$ & & & & \\
\hline
\end{tabular}

NA - information not available, $\mathrm{Y}$ - authors considered the cost component, but the proportion was unknown, EUR - Europe Region, AMR - The Americas Region, WPR - Western Pacific Region, SEAR - South East Asia, AFR - The Africa Region, EMR - Eastern Mediterranean Region

*Unpublished data.

$†$ Public health care.

¥Private health care.

§Pediatric ward.

\#Supplementary health system. 


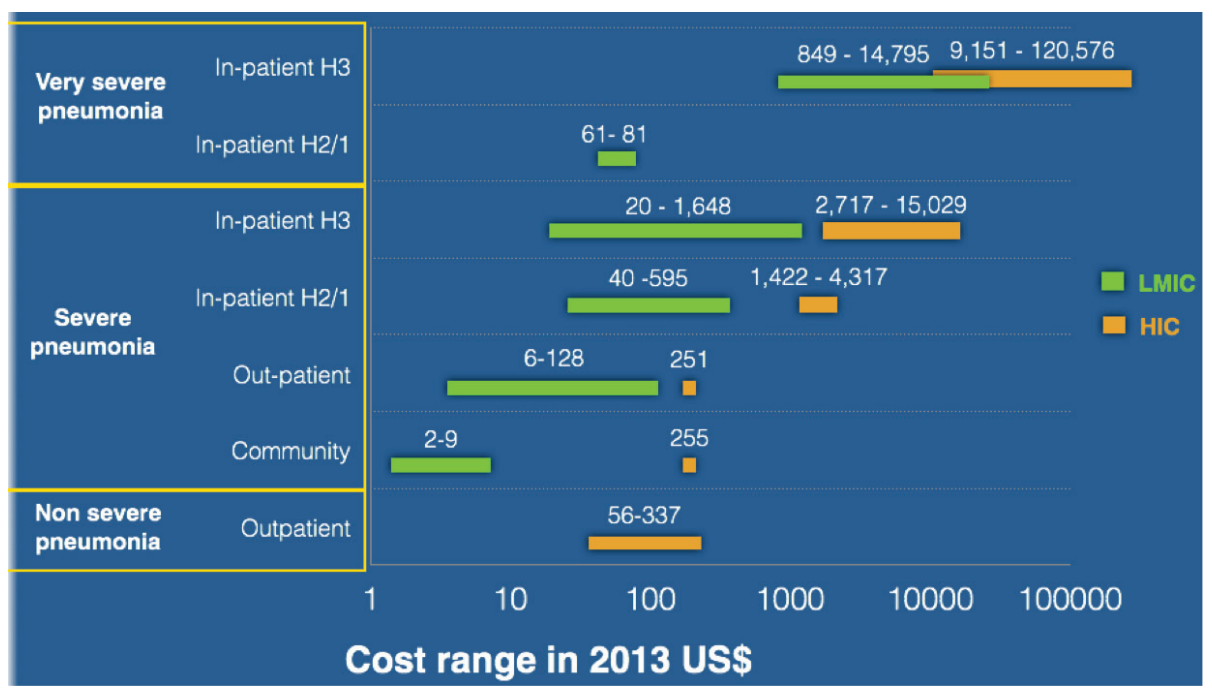

Figure 3. Range of cost per episode for pneumonia management (2013 US\$).

The costs for management of non-severe pneumonia managed at outpatient level were US\$ 66.9 (95\% CI 21.7129.7), which was slightly higher than for severe cases at outpatient level. This is because the hospital short stay for non-severe pneumonia in South Africa [22] was relatively high at US\$263.1 per episode. The mean cost for very severe cases was US\$ 6496.0 (2246.2-12 007.4), which is nearly 10 -fold of severe case management cost.

There were 6 studies reporting cost from household perspective, mainly from LMICs in South East Asia Region. Direct medical cost for severe pneumonia in hospital inpatient settings were $26.6 \%-115.8 \%$ of the monthly household income, thus demonstrating that severe pneumonia management in hospital placed a significant financial burden on families. On the other hand, outpatient and community management of severe pneumonia accounted for only $0.4 \%-4.1 \%$ of family's monthly income indicating decreased burden in these settings. (Table 4).

Of the papers reporting cost component of severe pneumonia management, direct medical cost was reported across all studies and accounted for $45 \%-100 \%$ of the total cost. The mean direct non-medical cost and indirect cost were US\$ 22.0 (11.8-32.7) and US\$ 27.0 (4.0-54.3) respectively, which account for $0.5 \%-31.0 \%$ of weekly household income (Table 5).

\section{Length of stay in hospital}

The in-patient cost was determined primarily by the length of stay (LOS) and the average cost per bed day. In this review, we extracted length of stay for severe pneumonia for future costing analysis reference (Tables 6-8).

Table 4. Direct medical cost for severe pneumonia management in low- and middle-income countries reported from household perspective

\begin{tabular}{|c|c|c|c|c|c|}
\hline \multirow[t]{2}{*}{ Country, pubucation year } & \multicolumn{4}{|c|}{ Direct medical cost (2013 \$US; \% OF DIRECt MEdical cost tO MONTHLY hOUSEHOLd InCOME) } & \multirow[t]{2}{*}{$\begin{array}{l}\text { MoNTHLY HOUSEHOLD } \\
\text { INCOME (2013 \$US)* }\end{array}$} \\
\hline & $\begin{array}{l}\text { Tertiary/teaching } \\
\text { Hospital in-patient care }\end{array}$ & $\begin{array}{c}\text { Secondary/primary } \\
\text { hospital in-patient care }\end{array}$ & Out-patient care & $\begin{array}{l}\text { Community } \\
\text { ambulatory care }\end{array}$ & \\
\hline Bangladesh, 2010 [26] & $124.2(52.9 \%)$ & - & - & - & 234.9 \\
\hline Bangladesh, 2005† & $80.6^{\ddagger}(34.3 \%)$ & $62.6^{\S}(26.6 \%)$ & & - & 234.9 \\
\hline India, $2009[30]$ & $305.8(115.8 \%)$ & $135.1(64.7 \%)$ & - & - & 264.0 \\
\hline Pakistan, 2012 [23] & - & - & $7.9(2.3 \%)$ & $1.5(0.4 \%)$ & 338.3 \\
\hline Viet Nam, 2001 [28] & - & - & - & $2.7(0.7 \%)$ & 398.6 \\
\hline Fiji, 2012 [34] & - & - & $25.7(4.1 \%) / 15.6(2.5 \%)$ & - & $632.5 \ddagger$ \\
\hline \multicolumn{6}{|c|}{$\begin{array}{l}\text { *Monthly household income ( } 2913 \text { US\$) were derived from Gallup World Poll annual median household income, equals annual median household } \\
\text { income divided by 12. These results were based on Gallup data gathered between } 2006 \text { and } 2012 \text { in } 131 \text { population. Source: http://www.gallup.com/ } \\
\text { poll/166211/worldwide-median-household-income-000.aspx. }\end{array}$} \\
\hline \multirow{2}{*}{\multicolumn{6}{|c|}{$\begin{array}{l}\dagger \text { Numbers used GNI per capita based on purchasing power parity (PPP) due to lack of monthly household income data. Source: http://data.worldbank. } \\
\text { org/indicator/NY.GNP.PCAP.PP.CD/countries. } \\
\text { †Public health care. }\end{array}$}} \\
\hline & & & & & \\
\hline \multicolumn{6}{|c|}{ §Supplementary health system. } \\
\hline
\end{tabular}


Table 5. Direct non-medical cost and indirect cost per episode for severe pneumonia management in low-and middle-income countries

\begin{tabular}{|c|c|c|c|c|c|c|}
\hline \multirow[t]{2}{*}{ Country, publication year } & \multicolumn{3}{|c|}{ DIRECT NON-MEDICAL COST ${ }^{*}$} & \multirow{2}{*}{$\begin{array}{l}\text { InDIRECT COSTt } \\
\text { Total }\end{array}$} & \multirow{2}{*}{$\begin{array}{l}\text { \% OF NON-MEDICAL COST TO } \\
\text { MONTHLY HOUSEHOLD INCOME }\end{array}$} & \multirow{2}{*}{$\begin{array}{l}\text { MONTHLY HOUSEHOLD } \\
\text { INCOME (2013 US\$) }\end{array}$} \\
\hline & Total & Transportation & Food & & & \\
\hline Bangladesh, 2010 [24] & 32.4 & & & & $13.8 \%$ & 234.9 \\
\hline India, $2002[29]$ & 5.3 & 5.3 & & 47.5 & $2.0 \%$ & 264.0 \\
\hline Pakistan, 2008 [19] & 35.9 & 12.2 & 23.7 & & $10.6 \%$ & 338.3 \\
\hline Pakistan, 2006 [20] & 55.0 & & & & $16.3 \%$ & 338.3 \\
\hline Pakistan, 2012 [23] & 3.3 & 2.3 & 1.0 & 9.6 & $1.0 \%$ & 338.3 \\
\hline Kenya, 2009 [32] & 14.0 & & & & $9.0 \%$ & 155.8 \\
\hline Guinea, 1998 [21] & 30.0 & & & & $31.0 \%$ & $96.7 \ddagger$ \\
\hline South Africa, 2012 [22] & 2.0 & 1.4 & & 0.2 & $0.5 \%$ & 434.8 \\
\hline Fiji, 2012 [34] & 33.2 & 33.2 & & 4.9 & $5.3 \%$ & $632.5 \ddagger$ \\
\hline Brazil, 2011* & 9.7 & 8.41 & 1.31 & 73.1 & $1.6 \%$ & 626.8 \\
\hline Mean $(95 \%$ CI) & $22.0(11.8-32.7)$ & $10.5(3.5-22.3)$ & $8.7(1.0-23.7)$ & $27.0(4.0-54.3)$ & & \\
\hline Median (IQR) & $22.0(4.6-33.9)$ & $6.9(2.1-11.5)$ & $1.3(-)$ & $9.6(2.5-60.3)$ & & \\
\hline
\end{tabular}

CI - confidence interval, IQR - interquartile range

*Direct medical costs include medications and consultation, non-medical cost includes transportation, food and accommodation.

$\dagger$ Indirect cost refers to parental loss of earnings in the period of illness. Monthly household income (2013 US\$) was derived from Gallup World Poll annual median household income, equals annual median household income divided by 12 . These results were based on Gallup data gathered between 2006 and 2012 in 131 populations. Source: http://www.gallup.com/poll/166211/worldwide-median-household-income-000.aspx.

\$Numbers used GNI per capita based on purchasing power parity (PPP) due to lack of monthly household income data. PPP GNI is gross national income (GNI) converted to international dollars using purchasing power parity rates. An international dollar has the same purchasing power over GNI as a US dollar has in the United States. GNI is the sum of value added by all resident producers plus any product taxes (less subsidies) not included in the valuation of output plus net receipts of primary income (compensation of employees and property income) from abroad. Source: http://data.worldbank. org/indicator/NY.GNP.PCAP.PP.CD/countries.

Table 6. Length of stay of very severe pneumonia and severe pneumonia in hospital in high-income countries

\begin{tabular}{|c|c|c|c|}
\hline Country and year & Setting & LOS (SD) DaYs & $\begin{array}{l}\text { SAMPLE } \\
\text { SIZE }\end{array}$ \\
\hline \multicolumn{4}{|c|}{ Very severe pneumonia: } \\
\hline Germany, 2005 [16] & ICU & $7.4(6.1)$ & 2039 \\
\hline Spain, 2013 [17] & ICU & 18.0 & 99 \\
\hline USA, 2012* & $\mathrm{ICU}$ & $18.3(43.1)$ & 1116 \\
\hline Australia, 2011* & ICU & 11.0 & - \\
\hline Median (IQR) & & $14.5(10.1-18.1)$ & \\
\hline \multicolumn{4}{|l|}{ Severe pneumonia: } \\
\hline \multirow[t]{2}{*}{ Ireland, 1999 [13] } & Control group & $8.3(7.7-9.0)$ & 44 \\
\hline & New treatment group & $4.0(3.5-4.6)$ & 45 \\
\hline Germany, 2005 [16] & Hospitalised cases & $7.4(6.1)$ & 2039 \\
\hline \multirow{2}{*}{ Australia, 2008 [14] } & Without impact diary & 8.8 & 202 \\
\hline & With impact diary & 13.5 & 523 \\
\hline Australia, 2011* & Non-ICU & 6.0 & - \\
\hline Spain, 2013 [17] & Non-ICU & 10.5 & 99 \\
\hline USA, 2012* & Non-ICU & $2.7(2.3)$ & 940 \\
\hline Median (IQR) & & $7.9(5.5-9.2)$ & \\
\hline
\end{tabular}

IQR - interquartile range, ICU - intensive care unit

*Unpublished data.

The mean LOS for severe pneumonia reported in individual studies ranged from 4-13.5 days, with a mean LOS 7.7 (95\% CI 5.5-9.9) days and median 7.9 (IQR 5.5-9.2) days in HIC, and mean LOS 5.8 (95\% CI IQR 5.3-6.4) days and median 6.4 (IQR 4.1-7.1) days in LMIC. For very severe pneumonia management in intensive care unit (ICU), LOS ranged from 7.4 to 18.3 days. The mean and median LOS were 13.7 (95\% CI IQR 9.2-18.2) and 14.5 (IQR 10.118.1) days in HIC, and 9.5 (95\%CI, 7.4-11.8) and 9.2 (IQR 6.1-12.6) days in LMIC.

\section{Unit cost of case management}

Unit cost of treatment and resource uptake should be routinely reported in cost studies. However, only 13 of the 34 included studies reported these data. Since treatment protocols (use of antibiotics, diagnostic tests, procedures and levels and intensity of care) varied between studies, this contributed to variations in costs across studies. For example, the average cost of chest radiograph in LMIC was US\$ 8.4 (95\% CI 4.3-27.0), which was significantly lower than US\$ 185.5 (95\% CI 66.3-357.7) in high income countries (Table 9). We attempted to abstract unit cost data but were unable to include it in the presented direct medical costs because of paucity of information.

\section{DISCUSSION}

This is the first attempt to conduct a systematic review of all published and available unpublished cost data on the management of childhood pneumonia. Costs per episodes in HICs were 5-13-fold higher in all delivery channels than those in LMICs. The review demonstrates that the magnitude of cost per episode increases markedly as the level of treatment delivery rises. Community management for severe pneumonia was less than $10 \%$ that of the cost of out-patient management among all levels of management in LMICs. Thus, there are strong economic reasons for considering community case management as a central strategy for pneumonia case management in low income countries; this merits further evaluation which should include consideration of medical outcomes. The mean lengths of stay 
Table 7. Length of stay of severe pneumonia in hospital in low- and middle-income countries

\begin{tabular}{|c|c|c|c|}
\hline Country, year & DEscRiPtION & LENGTH OF STAY (SD) IN DAYs & SAMPIE SIZE \\
\hline \multirow[t]{4}{*}{ Viet Nam, 2010 [18] } & Probable pneumonia & $7.2(5.0)$ & 40 \\
\hline & Radiograph confirmed & $6.7(3.8)$ & 426 \\
\hline & Probable severe pneumonia & $6.2(3.3)$ & 59 \\
\hline & Radiograph confirmed severe pneumonia & $6.4(2.7)$ & 193 \\
\hline Bangladesh, 2010 [24] & Hospital care & $6.0(5.0-7.0)$ & 180 \\
\hline \multirow[t]{2}{*}{ Bangladesh, 2005* } & Public health care & 7.1 & 73 \\
\hline & Private health care & 6.4 & 41 \\
\hline Bangladesh, 2010* & Hospital stay & $7.0(3.0) \dagger$ & 93 \\
\hline Pakistan, 2003 [25] & Antibiotic use duration & Approx. 8 & 124 \\
\hline \multirow[t]{7}{*}{ Kenya, 2009 [32] } & National hospitals & 8.2 & 49 \\
\hline & District hospitals & 6.7 & 30 \\
\hline & District hospitals & 4.8 & 29 \\
\hline & District hospitals & 4.2 & 17 \\
\hline & Provincial hospitals & 6.6 & 31 \\
\hline & Mission Hospitals & 7.8 & 30 \\
\hline & Mission Hospitals & 3.4 & 19 \\
\hline Zambia, 2009 [31] & Tertiary health center & 4.0 & 221 \\
\hline Pakistan, 2008 [19] & Time spent at health facility for severe pneumonia & 3.3 & 65 \\
\hline Pakistan, $2006[20]$ & Secondary hospital & 3.0 & 502 \\
\hline Jordan, 2010 [35] & In-patient days & $4.0-5.0$ & 728 \\
\hline \multirow[t]{2}{*}{ India, $2009[30]$} & Secondary hospital & $3.5(2.9-4.1)$ & 31 \\
\hline & Tertiary hospital & $3.7(3.0-4.4)$ & 25 \\
\hline India, 2002 [29] & Tertiary hospital & $6.5(2.5)$ & 52 \\
\hline \multirow{2}{*}{ Brazil, 2011* } & Public health system & $3.9(2.2)$ & 59 \\
\hline & Supplementary health system & $5.3(4.7)$ & 20 \\
\hline \multirow[t]{3}{*}{ Colombia, 2013 [27] } & Primary & $2.0(1.0-2.0)$ & 247 \\
\hline & Secondary hospital & $4.0(1.0-5.0)$ & 1208 \\
\hline & Tertiary hospital & $6.0(3.0-9.0)$ & 47 \\
\hline \multirow[t]{2}{*}{ South Africa, 2011 [33] } & Public sector ward & 8.7 & 86 \\
\hline & Fee for service sector & 5.6 & 7786 \\
\hline South Africa, 2012 [22] & Paediatric ward & $8.1(7.4-8.8)$ & 513 \\
\hline Indonesia, 2001* & Non-ICU & 6.7 & - \\
\hline \multirow[t]{3}{*}{ Argentina, 2012* } & Severe pneumonia & $7.5(8.5)$ & 42 \\
\hline & Unilateral focal pneumonia without complications & $7.4(6.0)$ & 1994 \\
\hline & Multifocal pneumonia without complications & $8.0(6.5)$ & 323 \\
\hline Median (IQR) & & $6.4(4.1-7.1)$ & \\
\hline
\end{tabular}

ICU - intensive care unit

*Unpublished data.

†Combined HIV+ and HIV-, HIV+ had longer stay in ward (9.3 vs 7.0 days).

¥Length of stay (LOS) reported as mean, mean (standard deviation) or median (interquartile range). When stratified LOS available, then stratified LOS was reported, not average length of stay of all pneumonia.

in hospital for severe pneumonia were 1.8-4.6 days less in LMIC compared to HIC, and at a mean of 5.8 and median of 6.4 days, were close to the WHO recommendation of 5 days in-patient treatment [10].

We demonstrated that the cost (per episode) for the management of severe pneumonia varied greatly by unit cost of intervention, disease severity and treatment procedures in different settings. The review also demonstrated that major factors governing the total cost per episode were length of stay in the hospital, countries income level and the presence or absence of community case management for pneumonia. Many other studies have also found GDP per capita to be the main driver of costs [37]. These findings demonstrate that choosing the appropriate value for these inputs will have a significant influence on the total cost. Existing studies calculated pneumonia management costs in many countries assuming the same treatment procedure and unit cost of medicine. However, the cost data we collected demonstrate that this method may have limitations; the uncertainty in the traditional estimates can be measured using the cost data reported in this review.

Our results showed that direct medical costs for childhood pneumonia management, especially inpatients, represent a significant proportion of the average monthly household income for families in LMICs. This is often compounded by further direct non-medical cost and indirect cost ie, loss 
Table 8. Length of stay of very severe and non-severe pneumonia in hospital in low- and middle-income countries

\begin{tabular}{|c|c|c|c|}
\hline CountRY, year & Deschiption & LENGTH OF STAY (SD) DAYS & Sample SIzE \\
\hline \multicolumn{4}{|l|}{ Very severe pneumonia } \\
\hline \multirow[t]{2}{*}{ Viet Nam, 2010 [18] } & Very severe pneumonia & $6.4(2.7)$ & 26 \\
\hline & Confirmed very severe pneumonia & $5.8(3.0)$ & 44 \\
\hline Colombia, 2013 [27] & ICU & $13.0(6.0-14.0)$ & 43 \\
\hline \multirow[t]{2}{*}{ South Africa, 2011 [33] } & $\mathrm{ICU}$ & 9.4 & 46 \\
\hline & $\mathrm{ICU}$ & 10.5 & 93 \\
\hline South Africa, 2012 [22] & ICU & $14.4(10.3-18.5)$ & 7 \\
\hline Pakistan, 2008 [19] & Time spent at health facility for very severe pneumonia & 3.9 & 35 \\
\hline \multirow[t]{3}{*}{ Argentina, $2012 \dagger$} & Very severe pneumonia & 8.9 & - \\
\hline & Unilateral focal pneumonia without complications & 17.2 & - \\
\hline & Multifocal pneumonia without complications & 11.5 & - \\
\hline \multirow[t]{2}{*}{ Brazil, 2011* } & Public health system & 6.9 & - \\
\hline & Supplementary health system & 6 & - \\
\hline Median (IQR) & & $9.2(6.1-12.6)$ & \\
\hline \multicolumn{4}{|l|}{ Non severe pneumonia } \\
\hline Pakistan, 2008 [19] & Time spent at health facility for pneumonia & 0.3 & 41 \\
\hline South Africa, 2012 [22] & Short stay & $1.4(1.3-1.6)$ & 338 \\
\hline Median (IQR) & & $0.9(0.3-1.4)$ & \\
\hline
\end{tabular}

ICU - intensive care unit, IQR - interquartile range

*Unpublished data.

$\dagger$ Note added in proof: The data from this study are unpublished but the data on the length of stay are published in Giglio ND, Cane AD, Micone P, Gentile A. Cost-effectiveness of the CRM-based 7-valent pneumococcal conjugated vaccine (PCV7) in Argentina. Vaccine. 2010;28:2302-10. Medline:20064478

Table 9. Chest Radiography cost per episode

\section{Countriv, YeAR}

COST PER EPISODE (US\$, 2013)

\begin{tabular}{|c|c|c|}
\hline \multirow{4}{*}{$\begin{array}{l}\text { High income } \\
\text { countries }\end{array}$} & Australia, 2011* & 129.8 \\
\hline & Chile, 2007 [12] & 135.1 \\
\hline & Uruguay, 2007 [12] & 43.4 \\
\hline & United States, 2012* & 433.7 \\
\hline Mean(SD) & & $185.5(66.3-357.7)$ \\
\hline Median(IQR) & & $132.5(108.2-209.8)$ \\
\hline \multirow{13}{*}{$\begin{array}{l}\text { Low- and } \\
\text { middle- } \\
\text { income } \\
\text { countries }\end{array}$} & Argentina, 2012* & 26.7 \\
\hline & Brazil, 2011* & 10.7 \\
\hline & & 6.0 \\
\hline & Brazil, 2007 [12] & 13.63 \\
\hline & Bangladesh, 2010* & 2.3 \\
\hline & India, 2009 [30] & 5.4 \\
\hline & Pakistan, 2008 [19] & 3.2 \\
\hline & Indonesia, 2001* & 4.6 \\
\hline & Kenya, 2009 [32] & 2.3 \\
\hline & South Africa, 2001* & 29.7 \\
\hline & South Africa, 2011 [33] & 59.7 \\
\hline & & 137.2 \\
\hline & South Africa, 2012 [22] & 27.7 \\
\hline Mean (SD) & & $25.3(9.8-47.3)$ \\
\hline Median (IQR) & & $8.4(4.3-27.0)$ \\
\hline
\end{tabular}

*Unpublished data.

of earnings when caring for the sick child. In countries where these families were uninsured, health payments for pneumonia management were a heavy burden on household and can have a significant impact on the family, particularly when the payments for care were out-of-pocket in most LMIC countries. Alamgir et al investigated the impact that this strain had on families and how they source the funds: many borrow or take high-interest loans [26]. Furthermore, Ayieko et al found that 10\% of the patients in district hospitals and up to $25 \%$ of children in tertiary hospitals wait in hospital beds after medical discharge while families source the fees. The latter translates to an additional cost of US\$ 17.46 to the public provider and US\$ 5.32 to the family [32], resulting in a drain on both the resources of the family and the health care provider, as well as denying a bed to another sick child. It is therefore important that national strategies for pneumonia management in LMIC are not only cost-effective for the national program but also give attention to the burden of costs on families so that these are maintained at a level that is affordable.

The data in this review comprise "actual" cost data measured in cost studies conducted in many LMIC and HIC. We believe that these represent a fair first approximation of true costs in these countries. It is noteworthy that the resulting cost estimates are higher than those currently contained in the WHO-CHOICE estimates [38]. Three factors could have contributed to this variation. First, we identified longer facility and hospital stays compared to standard treatment protocols recommended by the WHO [39]. Moreover, most existing cost studies were conducted at tertiary level hospitals where out-patient and in-patient treatments carry a much higher cost compared to the community or first level facility. Third, the wide variety of antibiotics (including variations in dosage, route of administration and duration) across the sites, as well as the heterogeneity in the costing methodology and the cost components in existing studies may have led to higher estimates. 
This review has several limitations. First, the primary goal of the systematic review was to obtain data on cost of management (per episode) of severe pneumonia. However, the lack of any standard management protocols (which varied widely across the included studies) and the general lack of service uptake data, may have contributed to the substantial uncertainty around the estimates. Second, we did not include costs of diagnostic investigations in the cost modeling in some study sites, because country-specific unit prices and utilization data were not available. Therefore, the true economic burden resulting from the management of childhood pneumonia could be considerably higher. Third, costs were highly dependent on level of care offered at facility and LOS could be skewed to longer period if high level of care (such as intensive care unit (ICU) care) was offered to severe and very severe cases. In this review, severe cases were all managed at non-ICU hospital settings, and very severe cases were managed at both non-ICU and ICU care in hospital settings. We were able to report LOS separately for non-ICU and ICU care but this stratification was not possible for total cost per episode. A further limitation was that the definition of ICU and ICU care may vary by country. Fourthly, we limited the search to English articles only, which may exclude some cost reported in other languages, however only 13 studies out of 789 articles in other languages were found. We tried to compliment this with unpublished data from non-English speaking countries. Lastly, there was a wide range in per capita income and health care system and payment schemes within LMIC category and the existing cost data may only reflect the situation when and where the data were collected and may not be representative of the whole country or the current situation. Furthermore, there were not sufficient studies to address all possible cost scenarios to facilitate international and public/private comparisons.
Our results demonstrate that further research on the economic burden due to the management of childhood pneumonia is needed, with clear reporting of data on unit cost of intervention, dosage of various drugs and information on health care utilization, such as length of stay in hospital. We recommend that standard reporting of unit cost of intervention with direct medical and non-medical costs and indirect costs, standard treatment protocols and health resource utilization in conjunction with the total cost per episode in any cost-of-illness studies would facilitate economic estimates of national scale-up and international comparisons. Further studies on the cost-effectiveness of standardized IMCI protocol against other treatment protocols could be expected to find a cost-saving management strategy for high burden countries.

Identifying the most cost-effective interventions for pneumonia management is essential for achieving the goal of further reducing child mortality. Our study demonstrated that early treatment in the community costs less (per event) than late treatment in the hospital. This finding suggests that the public health community should explore ways for community outreach for early diagnosis and treatment before severe pneumonia sets in. The results from this systematic review provide important missing information on the cost of pneumonia treatment in children across many countries. These data and the cost estimates should provide important information useful to program managers and policy makers at national and regional levels, international agencies, and donor organisations to aid resource allocation, program planning and priority setting. The estimates presented in this review could enable a more detailed economic evaluation of the revised WHO pneumonia management guidelines [39], and help identify the most cost-effective preventive and treatment interventions for reducing the burden of childhood pneumonia.

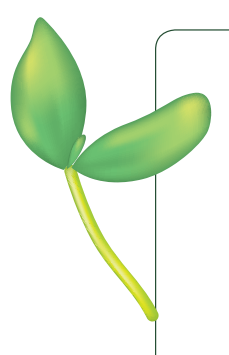

Acknowledgments: The authors thank the Maternal Neonatal Child and Adolescent Health (MNCAH) division of the WHO for funding the study and China Scholarship Council for the fellowship to SZ.

Funding: Shanshan Zhang is supported by WHO Maternal, Newborn, Child and Adolescent Health division and China Scholarship Council. Harish Nair and Harry Campbell received funding support from WHO Maternal, Newborn, Child and Adolescent Health division and Bill and Melinda Gates Foundation (OPP 1096225).

Authorship declaration: HN and HC conceived the study. SZ and HN designed the study. PMS, IK, SZ, CMT, SNA, AS, JKY, TMH, NG, MUB, BDG, PJCL, JAC, IA and RK performed the experiments. SZ analysed the data. SZ, HN and HC wrote the paper. AA, CMT, AS, SAM, GK, RB, SEA, QSR, AG, KSR, BDG, MN, PJCL, EAFS, ZAB and SBS critically reviewed the manuscript:. All authors have read, and confirm that they meet, ICMJE criteria for authorship.

Competing interests: HC is an editor-in-chief of the Journal of Global Health. To ensure that any possible conflict of interest relevant to the journal has been addressed, this article was reviewed according to best practice guidelines of international editorial organizations. All authors have completed the Unified Competing Interest form at www.icmje.org/coi_disclosure.pdf (available on request from the corresponding author). The authors have declared that no competing interests. 
1 Rudan I, O’Brien KL, Nair H, Liu L, Theodoratou E, Qazi S, et al. Epidemiology and etiology of childhood pneumonia in 2010: estimates of incidence, severe morbidity, mortality, underlying risk factors and causative pathogens for 192 countries. J Glob Health. 2013;3:010401. Medline:23826505

2 Liu L, Oza S, Hogan D, Perin J, Rudan I, Lawn JE, et al. Global, regional, and national causes of child mortality in 2000-13, with projections to inform post-2015 priorities: an updated systematic analysis. Lancet. 2015;385:430-40. Medline:25280870

3 Sazawal S, Black RE, Pneumonia Case Management Trials G. Effect of pneumonia case management on mortality in neonates, infants, and preschool children: a meta-analysis of community-based trials. Lancet Infect Dis. 2003;3:547-56. Medline:12954560 doi:10.1016/S1473-3099(03)00737-0

4 Bhutta ZA, Das JK, Walker N, Rizvi A, Campbell H, Rudan I, et al. Interventions to address deaths from childhood pneumonia and diarrhoea equitably: what works and at what cost? Lancet. 2013;381:1417-29. Medline:23582723 doi:10.1016/S0140-6736(13)60648-0

5 Rakha MA, Abdelmoneim A-NM, Farhoud S, Pičche S, Cousens S, Daelmans B, et al. Does implementation of the IMCI strategy have an impact on child mortality? A retrospective analysis of routine data from Egypt. BMJ Open. 2013;3:e001852. Medline:23355663 doi:10.1136/bmjopen-2012-001852

6 Nair H, Simőes EA, Rudan I, Gessner BD, Azziz Baumgartner E, Zhang JSF, et al. Global and regional burden of hospital admissions for severe acute lower respiratory infections in young children in 2010: a systematic analysis. Lancet. 2013;381:1380-90. Medline:23369797 doi:10.1016/S0140-6736(12)61901-1

7 Bahia L, Toscano CM, Takemoto MLS, Araujo DV. Systematic review of pneumococcal disease costs and productivity loss studies in Latin America and the Caribbean. Vaccine. 2013;31S:C33-44. Medline:23777689 doi:10.1016/j.vaccine.2013.05.030

8 WorldBank. Country and lending groups 2014. Available: http://data.worldbank.org/about/country-and-lending-groups\#high_income. Accessed: 22 October 2014.

9 Drummond M, Sculpher M, Torrance G, O'Brien B, Stoddart G. Critical assessment of economic evaluation. 3rd edition. Oxford: Oxford University Press; 2005.

10 WHO. Hospital care for children: World Health Organization; 2005

11 Gallup. Worldwide median household income 2013. Available: http://www.gallup.com/poll/166211/worldwidemedian-household-income-000.aspx. Accessed: 13 February 2015.

12 Constenla D. Evaluating the costs of pneumococcal disease in selected Latin American countries. Rev Panam Salud Publica. 2007;22:268-78. Medline:18078589 doi:10.1590/S1020-49892007000900007

13 Al-Eidan FA, McElnay JC, Scott M, Kearney M, Troughton K, Jenkins J. Sequential antimicrobial therapy: treatment of severe lower respiratory tract infections in children. J Antimicrob Chemother. 1999;44:709-15. Medline:10552992 doi:10.1093/jac/44.5.709

14 Lambert SB, Allen KM, Carter RC, Nolan TM. The cost of community-managed viral respiratory illnesses in a cohort of healthy preschool-aged children. Respir Res. 2008;9:11. Medline:18215329 doi:10.1186/1465-9921-9-11

15 Newall AT, Scuffham PA. Influenza-related disease: the cost to the Australian healthcare system. Vaccine. 2008;26:6818-23. Medline:18940222 doi:10.1016/j.vaccine.2008.09.086

16 Ehlken B, Ihorst G, Lippert B, Rohwedder A, Petersen G, Schumacher M, et al. Economic impact of community-acquired and nosocomial lower respiratory tract infections in young children in Germany. Eur J Pediatr. 2005;164:607-15. Medline:15965766 doi:10.1007/s00431-005-1705-0

17 Brotons P, Gelabert G, Launes C, Sicuri E, Pallares R, Munoz-Almagro C. Cost of hospitalizing children with invasive pneumococcal pneumonia. Vaccine. 2013;31:1117-22. Medline:23262168 doi:10.1016/j.vaccine.2012.12.025

18 Anh DD, Riewpaiboon A, Le Huu Tho S, Kim BN, Kilgore P. Treatment costs of pneumonia, meningitis, sepsis, and other diseases among hospitalized children in Viet Nam. J Health Popul Nutr. 2010;28:436-42. Medline:20941894

19 Hussain H, Waters H, Khan AJ, Omer SB, Halsey NA. Economic analysis of childhood pneumonia in Northern Pakistan. Health Policy Plan. 2008;23:438-42. Medline:18755733 doi:10.1093/heapol/czn033

20 Hussain H, Waters H, Omer SB, Khan A, Baig IY, Mistry R, et al. The cost of treatment for child pneumonias and meningitis in the Northern Areas of Pakistan. Int J Health Plann Manage. 2006;21:229-38. Medline:17044548 doi:10.1002/hpm.847

21 Jha P, Bangoura O, Ranson K. The cost-effectiveness of forty health interventions in Guinea. Health Policy Plan. 1998;13:249-62. Medline:10187595 doi:10.1093/heapol/13.3.249

22 Sinha A, Kim S, Ginsberg G, Franklin H, Kohberger R, Strutton D, et al. Economic burden of acute lower respiratory tract infection in South African children. Paediatr Int Child Health. 2012;32:65-73. Medline:22595212 doi:10.1179/2046905512Y.0000000010

23 Sadruddin S, Shehzad S, Bari A, Khan A, Khan A, Qazi S. Household costs for treatment of severe pneumonia in Pakistan. Am J Trop Med Hyg. 2012;87:137-43. Medline:23136289 doi:10.4269/ajtmh.2012.12-0242

24 Ashraf H, Mahmud R, Alam NH, Jahan SA, Kamal SM, Haque F, et al. Randomized controlled trial of day care versus hospital care of severe pneumonia in Bangladesh. Pediatrics. 2010;126:e807-15. Medline:20855397 doi:10.1542/peds.2009-3631

25 Aurangzeb B, Hameed A. Comparative efficacy of amoxicillin, cefuroxime and clarithromycin in the treatment of community-acquired pneumonia in children. J Coll Physicians Surg Pak. 2003;13:704-7. Medline:15569557 
26 Alamgir NI, Naheed A, Luby S. Coping strategies for financial burdens in families with childhood pneumonia in Bangladesh. BMC Public Health. 2010;10:622. Medline:20955627 doi:10.1186/1471-2458-10-622

27 Alvis-Guzman N, Orozco-Africano J, Paternina-Caicedo A, Coronell-Rodríguez W, Alvis-Estrada L, Jervis-Jálabe D, et al. Treatment costs of diarrheal disease and all-cause pneumonia among children under-5 years of age in Colombia. Vaccine. 2013;31:C58-62. Medline:23777692 doi:10.1016/j.vaccine.2013.05.035

28 Toan NV, Khe ND, Hojer B. Expenditure and payment sources for treating acute respiratory infections in rural Vietnam. Southeast Asian J Trop Med Public Health. 2001;32:682-8. Medline:12041539

29 Sahu S, Satapathy D, Sahu T, Tripathy R, Das B, Pradhan S. A study of acute respiratory tract infection cases admitted to a tertiary level health centre. Health and Population. 2002;25:186-93.

30 Madsen HO, Hanehřj M, Das AR, Moses PD, Rose W, Puliyel M, et al. Costing of severe pneumonia in hospitalized infants and children aged 2-36 months, at a secondary and tertiary level hospital of a not-for-profit organization. Trop Med Int Health. 2009;14:1315-22. Medline:19719464 doi:10.1111/j.1365-3156.2009.02374.x

31 Chola L, Robberstad B. Estimating average inpatient and outpatient costs and childhood pneumonia and diarrhoea treatment costs in an urban health centre in Zambia. Cost Eff Resour Alloc. 2009;7:16. Medline:19845966 doi:10.1186/1478-7547-7-16

32 Ayieko P, Akumu AO, Griffiths UK, English M. The economic burden of inpatient paediatric care in Kenya: household and provider costs for treatment of pneumonia, malaria and meningitis. Cost Eff Resour Alloc. 2009;7:3. Medline:19161598 doi:10.1186/1478-7547-7-3

33 Kitchin OP, Wessels F, Masekela R, Becker P, Green RJ. Costs of admission for paediatric pneumonia in a setting of human immunodeficiency virus infection. Int J Tuberc Lung Dis. 2011;15:1702-7. Medline:22118183 doi:10.5588/ijtld.11.0167

34 Temple B, Griffiths UK, Mulholland EK, Ratu FT, Tikoduadua L, Russell FM. The cost of outpatient pneumonia in children<5yearsofagein Fiji. TropMed IntHealth. 2012;17:197-203. Medline:22008519doi:10.1111/j.1365-3156.2011.02897.x

35 Khuri-Bulos N, Williams JV, Shehabi AA, Faouri S, Jundi EA, Abushariah O, et al. Burden of respiratory syncytial virus in hospitalized infants and young children in Amman, Jordan. Scand J Infect Dis. 2010;42:368-74. Medline:20100116 doi:10.3109/00365540903496544

36 Carosone-Link P, Simoes E, Child J. Cost of management of severe pneumonia in young children in Colorado. 2014. Unpublished data.

37 Adam T, Evans D, Murray C. Econometric estimation of country-specific hospital costs. Cost Eff Resour Alloc. 2003;1:3. Medline:12773218 doi:10.1186/1478-7547-1-3

38 Cost effectiveness and strategic planning (WHO-CHOICE).-Quantities and unit prices (cost inputs). Available: http://www.who.int/choice/cost-effectiveness/inputs/en/. Accessed: 22 October 2014.

39 WHO. Pocket book of hospital care for children. WHO 2013. Available: http://apps. who.int/iris/bitstre am/10665/81170/1/9789241548373_eng.pdf. Accessed: 22 October 2014. 